\title{
Asynchronisme du développement du tractus génital de l'escargot Helix aspersa pendant la croissance et la reproduction
}

\author{
J Bride, L Gomot \\ URA CNRS 687, faculté des sciences, laboratoire de zoologie et embryologie, \\ place Maréchal Leclerc, 25030 Besançon Cedex, France
}

(Reçu le 18 décembre 1989; accepté le 7 décembre 1990)

\begin{abstract}
Résumé - Pendant la croissance d'Helix aspersa en laboratoire, le développement de la glande à albumen et du spermoviducte est examiné en relation avec l'âge, le poids, le rebord de la coquille et les stades de la reproduction (accouplement et ponte). Les résultats montrent que la population peut être divisée en 2 sous-ensembles dont l'évolution est caractérisée par des taux de croissance pondérale différents et par l'asynchronisme du développement du tractus génital. Les escargots à croissance rapide arrivent les premiers à l'état adulte et sont de plus grande taille que ceux à croissance lente. Les premiers présentent un murissement précoce du type femelle, les autres donnent des adultes au stade mâle. Au cours de la croissance, la maturation du tractus génital n'a pas un rapport direct avec le poids des animaux. Dans chaque classe de même âge et de même poids, on peut distinguer 2 groupes d'animaux : la moitié des escargots possède un tractus significativement moins évolué que l'autre. L'asynchronisme du développement génital est encore présent au moment de l'accouplement. En effet, chez des partenaires sexuels de même poids, le tractus génital peut être jusqu'à 3 fois moins développé chez l'un que chez l'autre. Ces observations suggèrent que l'asynchronisme dans le développement de l'appareil génital serait une stratégie caractéristique de l'évolution de la population chez Helix aspersa. La disparition des différences entre les appareils génitaux des escargots après l'accouplement suggère aussi un rôle régulateur de l'accouplement.
\end{abstract}

\section{Helix aspersa / asynchronisme / tractus génital / glande à albumen / accouplement}

Summary - Asynchronous development of the genital tract during growth and reproduction in the snail, Helix aspersa. During the growth of laboratory-raised Helix aspersa, the development of the albumen gland and spermoviduct was studied in relation to age, weight and different stages of reproduction such as mating and egg-laying. The results showed that the population can be divided into 2 categories because of different growth rates and asynchronous development of the genital tract. The fast-growing snails are larger and reach sexual maturity (female type maturity) in a shorter period than slow growing snails (male type maturity). During growth, the maturation of the genital tract was not directly proportional to the weight of the animal. Within each class of similar weight and age of population, 2 groups were identified; in one group, the genital tract was significantly less developed than in the other. This asynchrony of genital tract development could still be found between mating partners of the same weight, the genital tract could be 3 times smaller in one than in the other partner. However, this difference disappeared between mating and egg-laying. These observations suggest:

- that asynchronous development of the genital tract could be a strategy for the developing population of Helix aspersa; and

- that (a) mating factor(s) is (are) involved in the regulation of the growth and function of the genital tract. 


\section{INTRODUCTION}

Le développement et le fonctionnement de l'appareil génital des Mollusques Gastéropodes ont fait l'objet de nombreux travaux analysés par Tompa (1984) et Geraerts et Joose (1984) et leur contrôle endocrine a été démontré (revue par Joosse et Geraerts, 1984). Chez Helix aspersa, l'évolution chronologique des étapes successives de la différenciation des conduits génitaux et de leurs organes accessoires a été observée dans des conditions d'environnement semi-artificielles (Courtot et Gomot, 1982; Enée et Griffond, 1983; Enée et Gomot, 1984) et en relation avec les phases de croissance d'une population (Gomot et Enée, 1980). Après la mise au point de conditions d'élevage complètement artificielles et contrôlées (Gomot et Deray, 1987), nous avons entrepris de nouvelles observations car l'effet de l'environnement sur la croissance et la reproduction d'Helix aspersa est important (Charrier, 1980; Enée et al, 1982; Gomot et al, 1982; Lucarz et Gomot, 1984, 1985; Bonnefoy-Claudet et Deray, 1987), comme chez d'autres escargots terrestres (revue par Goodfriend, 1986) ou aquatiques (revue par Calow, 1983).

L'objectif principal de cette étude était de déterminer les critères permettant d'évaluer avec précision les stades de maturation du tractus génital dont l'homogénéité est une condition indispensable à la reproductibilité des résultats expérimentaux, aussi bien in vivo qu'in vitro. Les observations du tractus ont donc été réalisées en corrélation avec les critères d'évolution de la population (âge, croissance pondérale, formation du rebord de la coquille) et les stades de la reproduction (accouplement et ponte).

\section{MATÉRIEL ET MÉTHODES}

\section{Les animaux}

Afin d'obtenir une population aussi identique que possible à un groupe naturel, le lot étudié est composé de 100 escargots nés au même moment de 3 pontes (génération $F_{1}$ ). lls ont été élevés au centre universitaire d'héliciculture $(\mathrm{CUH})^{*}$, à raison de 25 animaux par cage autonettoyante $\left(0,5 \mathrm{~m}^{2}\right)$, sous une photopériode fixe de $18 \mathrm{~h}$ de lumière par jour et à une température constante de $20^{\circ} \mathrm{C}$. L'humidité est maintenue à $95 \%$. L'aliment en farine (UCAAB*) pour escargot est distribué ad libitum le matin après le nettoyage. Les pontes proviennent d'escargots adultes ramassés dans la nature au début de leur hibernation et gardés en inactivité pendant 6 mois au $\mathrm{CUH}$ à $5^{\circ} \mathrm{C}$, avant d'être remis en activité dans les conditions d'environnement décrites ci-dessus qui sont également favorables à la reproduction.

\section{Étude de l'évolution pondérale des animaux}

À partir de 2,5 mois, les escargots sont pesés individuellement chaque semaine et l'histogramme des fréquences de poids est établi après répartition des animaux en classes différant entre elles de $1 \mathrm{~g}$.

\section{Prélèvement d'organes}

\section{Période de croissance}

En fonction d'observations préliminaires ayant permis d'établir une corrélation approximative entre l'âge, le profil de l'histogramme de fréquence de poids, la morphologie du péristome et le stade de différenciation du tractus génital, les animaux sont choisis dans la classe de poids la plus représentative pour le stade sexuel recherché (tableau I).

\footnotetext{
- Centre universitaire d'héliciculture, 5, rue Ronchaux, 25000 Besançon, France
} 
Pour l'observation de tractus génitaux immatures, un prélèvement d'escargots non bordés (nb) a été effectué à 3 mois (fig 1a) dans la classe de poids de 5-6 g qui ne contient pas d'animaux adultes caractérisés par l'épaississement du rebord de la coquille (escargots bordés: b).

Afin de cerner le passage entre le stade immature et le stade adulte, des escargots ont été prélevés à 4 mois (fig 1b) dans la classe de poids de $8-9 \mathrm{~g}$ où les animaux non bordés (nb) immatures et bordés (b) adultes sont présents dans des proportions voisines.

Des animaux adultes sont également prélevés dans la classe de poids de 10-11 g (fig 1b) où les immatures $(n b)$ sont en minorité.

\section{Période de reproduction}

\section{À l'accouplement}

Au début de la période d'accouplement de la génération $F_{1}$ (à partir de 4 mois), le tractus génital est prélevé au moment de la séparation des partenaires qui copulent pour la première fois $(10$ couples). Dans le lot des géniteurs (parents de $F_{1}$ ) remis en activité après hibernation (tableau I), les 4 premiers couples ont également été étudiés. Des prélèvements ont aussi été effectués au hasard pendant la période de reproduction d'autres générations $F_{1}$ élevées dans les mêmes conditions (26 couples) et d'autres lots d'adultes remis en activité après hibernation (8 couples).

\section{Au début de la période de ponte}

Des pots remplis de terre sont déposés dans les cages; les 15 premiers escargots observés en position de ponte sont étudiés. Parmi eux, 6 viennent de terminer de pondre, tandis que 9 escargots n'ont pas encore commencé à former. leurs œufs et ont stocké leurs ovocytes dans la chambre de fécondation avant la libération de la sécrétion de la glande à albumen.

\section{Méthode de détermination des stades de développement du tractus}

Nos recherches expérimentales portant particulièrement sur la glande à albumen et le spermoviducte, ce sont ces organes qui ont été principalement examinés ici.

\section{Observation morphologique}

Pour des raisons pratiques, nous avons utilisé les critères morphologiques décrits par Gomot et Enée (1980) pour caractériser les différents stades : infantile (I), juvénile (J), pré-adulte (PA), adulte (A). Le stade juvénile a été divisé en 3 étapes: $J_{1}$ lorsque le spermoviducte apparaît sous la forme d'un tube lisse séparé par 2 plis en une goutière mâle (spermiducte + ébauche prostatique) et une gouttière femelle (oviducte), $\mathrm{J}_{2}$ lorsque la paroi de l'oviducte commence à montrer des plis transversaux (aspect festonné), $\mathrm{J}_{3}$ lorsque l'épaississement de la paroi de l'oviducte à l'extrémité proximale indique le début de son activité glandulaire.

\section{Étude morphométrique du spermoviducte}

Afin de quantifier les observations, un fragment du spermoviducte est prélevé dans sa partie moyenne (fig 4) et inclu en paraffine. La surface transversale du spermoviducte à chaque stade de maturation est évaluée sur 3 coupes histologiques provenant de 3 animaux, à l'aide d'un analyseur d'images «Kontron MOP 3 ".

\section{Étude pondérale des organes du tractus}

L'indice de maturation (im) de la glande à albumen est obtenu par le rapport : poids de la glande/poids de l'animal $\times 100$. L'im du spermoviducte est évalué selon la même formule chez

* UCAAB, Chierry, 02400 Chateau-Thierry, France 
les animaux accouplés. L'im des composants du spermoviducte (prostate et oviducte) est déterminé par le rapport : poids de l'organe/poids du spermoviducte $\times 100$.

\section{Dosage du galactogène dans la glande à albumen}

Une partie de la glande est prélevée après la dissection et pesée dans un tube d'Eppendorf où est ajouté $1 \mathrm{ml}$ de $\mathrm{KOH} \mathrm{2,5} \mathrm{N}$. Les polysaccharides sont extraits selon la méthode de Van Handel (1965). La quantité de galactogène est évaluée par dosage enzymatique en présence de $\beta$-galactose deshydrogénase (Boehringer) après hydrolyse acide (Joosse et Van Elk, 1986).

\section{Analyse statistique des résultats}

Les moyennes sont comparées selon le test de Newman et Keul (Sokal et Rolf, 1969) précédé d'une analyse de variance, $P=0,05$.

\section{RÉSULTATS}

\section{Étude pondérale des animaux}

\section{Pendant la croissance}

Les histogrammes des fréquences de poids montrent la répartition des animaux (fig 1).

\section{À 3 mois (fig 1a)}

Le nombre des immatures dont le poids varie entre 1 et $11 \mathrm{~g}$ est supérieur à celui des individus adultes dont le poids est compris entre 6 et $12 \mathrm{~g}$. L'histogramme de croissance montre 2 pics : I'un dans la classe de $6-7 \mathrm{~g}$ (poids minimal des escargots adultes bordés, où les immatures non bordés sont les plus nombreux, et l'autre dans la classe de 10 à $11 \mathrm{~g}$ où les animaux adultes sont en majorité.

\section{À 4 mois (fig 1b)}

La croissance de la population se manifeste par l'augmentation du nombre d'animaux répartis dans les classes supérieures à $7 \mathrm{~g}$ et par leur diminution dans les classes inférieures à $7 \mathrm{~g}$. Le premier pic s'est déplacé dans la classe de 8-9 g où les immatures, dont le poids a augmenté, représentent encore 2/5 des individus. Le deuxième pic est toujours observé dans la classe de $10-11 \mathrm{~g}$ où le nombre d'animaux adultes a augmenté. L'évolution de la maturation sexuelle est indiquée par l'augmentation importante du nombre d'animaux bordés dans les classes supérieures à $7 \mathrm{~g}$, mais l'apparition d'individus bordés, donc adultes, dans la classe de 5$6 \mathrm{~g}$ suggère un blocage ou ralentissement de la croissance dans les classes inférieures à $7 \mathrm{~g}$, aux environs de 4 mois. Par contre, le poids des escargots immatures à croissance rapide a augmenté jusqu'à $12 \mathrm{~g}$.

\section{À l'accouplement (tableau I)}

Les jeunes adultes qui s'accouplent ont un poids irrégulier car la moyenne pondérale des 20 animaux est accompagnée d'un écart type important $(10 \mathrm{~g} \pm 2,6 \mathrm{~g})$. Les couples sont constitués de 2 animaux, de type 1 et de type 2, différents par le développement de leur appareil génital (fig 4). Bien que le poids des individus de type 1 soit légèrement supérieur, la différence entre les moyennes pondérales des 2 catégories (tableau I) n'est pas statistiquement significative.

Dans le lot des géniteurs (parents de $\left.F_{1}\right)$, le poids moyen des 8 animaux $(11,9 \mathrm{~g}$ $\pm 0,4 \mathrm{~g})$ n'est pas significativement différent de celui des jeunes adultes de $F_{1}$. De même, la différence entre les poids 
non bordes (immatures, nb) bordés (maturation sexuelle, b)

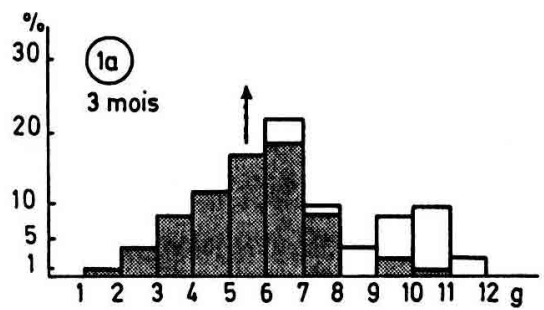

$\longrightarrow$ prélèvement déscargots non bordés prélevement d'escargots bordés

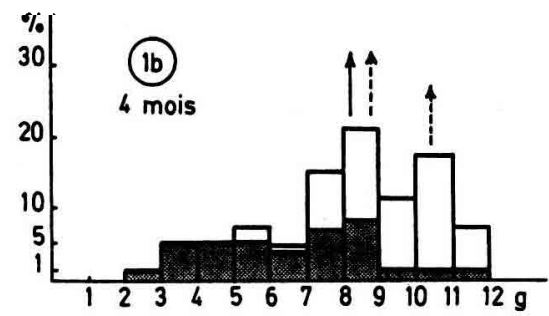

Fig 1. Histogramme de répartition des animaux en \% par classes de poids à 3 mois (1a) et à 4 mois (1b).

Tableau I. Relation entre les stades de développement du tractus génital $\left(J_{1}, J_{2}, J_{3}\right.$ : juvéniles; PA : pré-adulte; A : adulte), le poids de la glande à albumen, les caractères externes (âge, poids du corps, coquille non bordée : $\mathrm{nb}$ ou bordée : b), les stades de l'évolution sexuelle (immature, transition, adulte, accouplement).

\begin{tabular}{|c|c|c|c|c|c|c|c|}
\hline \multirow{2}{*}{$\begin{array}{l}\text { Stade } \\
\text { sexuel }\end{array}$} & \multirow[b]{2}{*}{ âge } & \multicolumn{3}{|c|}{ Paramètres externes } & \multicolumn{2}{|c|}{$\begin{array}{l}\text { Stades du } \\
\text { tractus génital }\end{array}$} & \multirow{2}{*}{$\begin{array}{l}\text { Poids de la } \\
\text { glande a } \\
\text { albumen (g) }\end{array}$} \\
\hline & & $\begin{array}{l}\text { Classe de } \\
\text { poids }(g)\end{array}$ & Coquille & $\begin{array}{l}\text { Nombre } \\
\text { d'animaux }\end{array}$ & Nombre & Taux & \\
\hline \multicolumn{8}{|c|}{ Croissance de $F_{1}$} \\
\hline Immature & 3 mois & $5-6$ & $\mathrm{nb}$ & 10 & $\begin{array}{ll}4 & J_{1} \\
6 & J_{2}\end{array}$ & $\begin{array}{l}40 \% \\
60 \%\end{array}$ & $\begin{array}{rr}8,5 \pm & 7 \\
106 \pm & 31\end{array}$ \\
\hline \multirow[b]{2}{*}{ Transition } & & & $\mathrm{nb}$ & 7 & $\begin{array}{ll}4 & J_{2} \\
3 & J_{3}\end{array}$ & $\begin{array}{l}57 \% \\
43 \%\end{array}$ & $\begin{array}{l}139 \pm 100 \\
368 \pm 194\end{array}$ \\
\hline & 4 mois & $8-9$ & b & 7 & $\begin{array}{ll}3 & J_{3} \\
4 & \mathrm{PA}\end{array}$ & $\begin{array}{l}43 \% \\
57 \%\end{array}$ & $\begin{array}{l}332 \pm 77 \\
752 \pm 86\end{array}$ \\
\hline Adulte & 4 mois & $10-11$ & $b$ & 7 & $\begin{array}{ll}2 & \text { PA } \\
5 & A\end{array}$ & $\begin{array}{l}29 \% \\
71 \%\end{array}$ & $\begin{array}{r}873 \pm 94 \\
1266 \pm 56\end{array}$ \\
\hline Accouplement & Type 1 & $10,3 \pm 2,5$ & $\begin{array}{l}b \\
b\end{array}$ & $\begin{array}{l}10 \\
15,4\end{array}$ & $\begin{array}{ll}9 & P A \\
1 & P A\end{array}$ & $\begin{array}{l}90 \% \\
10 \%\end{array}$ & $1022 \pm 292$ \\
\hline$r_{1}$ & type 2 & $9,6 \pm 2,8$ & $\begin{array}{l}b \\
b\end{array}$ & 10 & $\begin{array}{ll}9 & J_{3} \\
1 & P A\end{array}$ & $\begin{array}{l}90 \% \\
10 \%\end{array}$ & $376 \pm 119$ \\
\hline $\begin{array}{l}\text { Parents } \\
\text { de } F_{1}\end{array}$ & $\begin{array}{l}\text { type } 1 \\
\text { type } 2\end{array}$ & $\begin{array}{l}12,4 \pm 1 \\
11,4 \pm 0,9\end{array}$ & $\begin{array}{l}b \\
b\end{array}$ & $\begin{array}{l}4 \\
4\end{array}$ & & & $\begin{array}{l}926 \pm 121 \\
250 \pm 66\end{array}$ \\
\hline
\end{tabular}


moyens des individus de type 1 et de type 2 (tableau I) n'est pas statistiquement significative.

\section{Développement du tractus génital}

\section{Observation macroscopique générale et étude pondérale de la glande à albu- men}

\section{Période de croissance}

Le diagramme de la fig 2 et le tableau I montrent des variations individuelles dans le développement du tractus génital des escargots ayant le même âge et le même poids, et ceci, tout au long de la croissance du lot. Par exemple, à 3 mois, dans la classe de poids de 5-6 g, tous les es- cargots sont immatures et ont un tractus génital, soit au stade $J_{1}(40 \%)$ soit au stade $\mathrm{J}_{2}(60 \%)$. À 4 mois, dans la classe de $8-9 \mathrm{~g}$, les escargots encore immatures sont au stade $\mathrm{J}_{2}(57 \%)$ ou au stade $\mathrm{J}_{3}$ $(43 \%)$, tandis que les bordés adultes atteignent le stade PA $(57 \%)$, mais avec persistance du stade $J_{3}(43 \%)$. Par contre, dans une classe de poids supérieure (10$11 \mathrm{~g}$ ), le tractus génital des adultes est au stade $\mathrm{A}(71 \%)$ et le taux d'animaux en retard (stade PA), a diminué (29\%).

Dans chaque classe de poids de même âge, les différences anatomiques globales des tractus correspondent à des différences pondérales des glandes à albumen (tableau I) caractérisées par des im (poids relatifs) moyens différents qui permettent de les répartir en 2 stades par classe de poids (fig 3 ). Cependant, le rapport entre

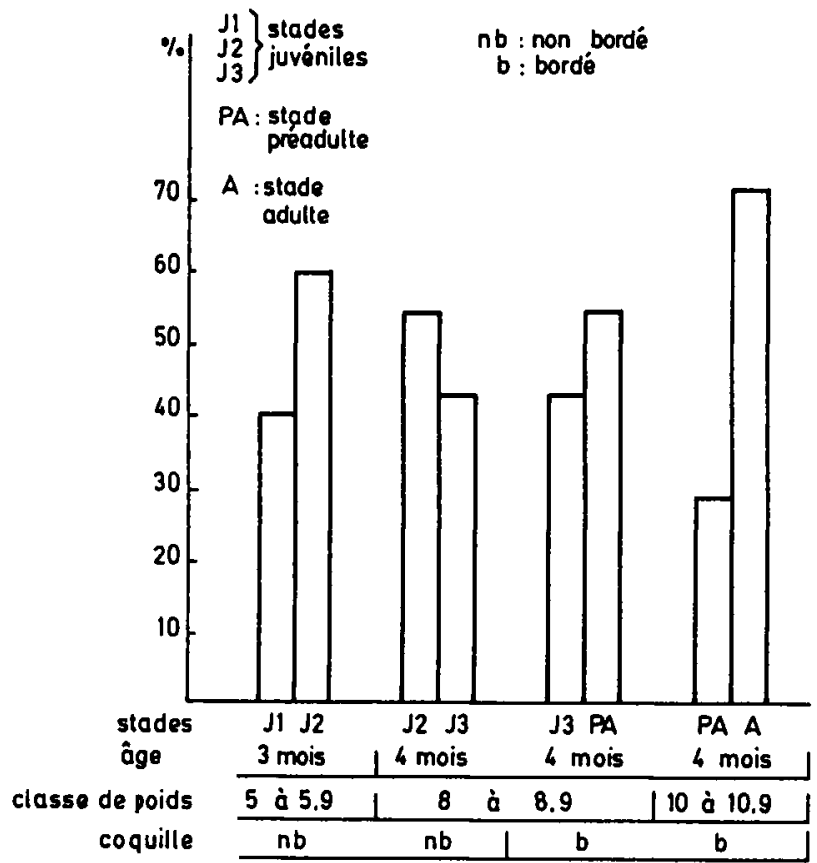

Fig 2. Histogramme de répartition des stades de développement du tractus génital au cours de la croissance des animaux. 
les poids moyens des 2 catégories de glandes à albumen de chaque classe d'âge diminue au fur et à mesure de la croissance (R, fig 3$)$.

\section{Périodes de reproduction}

A l'accouplement, chez les jeunes adultes de la génération $F_{1}$ étudiée depuis la nais- sance, les partenaires ont un tractus génital dont le développement inégal apparaît dans la fig 4 : le partenaire de type 1 possède une volumineuse glande à albumen et un spermoviducte très développé (stade A). Par contre, le tractus génital est moins développé chez le partenaire de type 2: de 1/3 pour le poids de la glande à albumen et presque de $1 / 2$ pour le spermoviducte

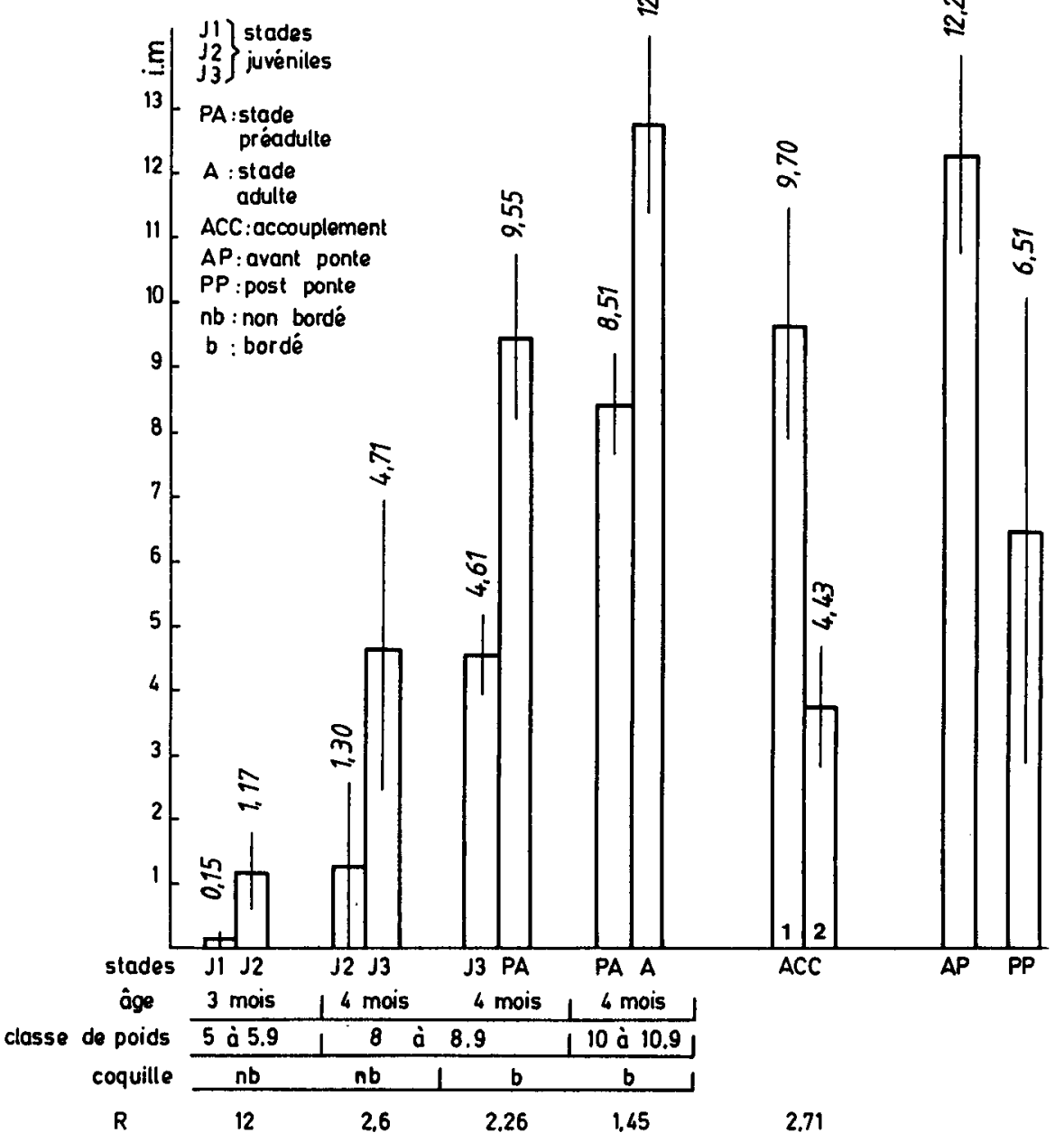

Fig 3. Evolution de l'indice de maturation (im) de la glande à albumen pendant la croissance, à l'accouplement et à la ponte. $R$ = rapport des poids moyens des 2 catégories de glande à albumen dans chaque classe de poids. 


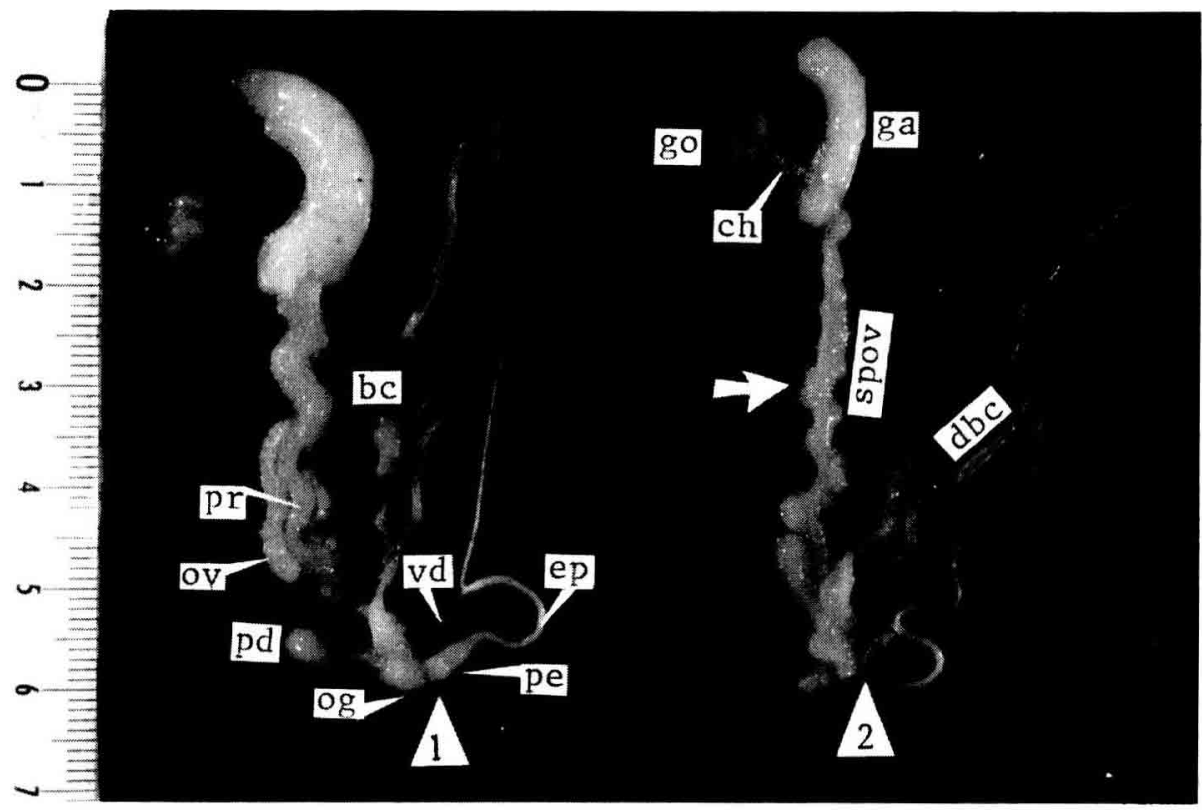

Fig 4. Appareil génital des partenaires (1 et 2) d'un couple. bc: bourse copulatrice; ch: canal hermaphrodite; dbc: diverticule de la bourse copulatrice; ep: épiphalus; ga: glande à albumen; go: gonade; og: orifice génital; ov: oviducte; pd: poche du dard; pe: pénis; pr: prostate; spov: spermoviducte; vd: vas deferens. La flèche montre la zone prélevée pour l'étude histologique.

d'après l'analyse morphométrique de la surface des coupes transversales (fig 5).

Pour les 10 couples étudiés, le poids moyen des glandes de type 2 (tableau I) et l'indice moyen (fig 3 ) sont équivalents aux valeurs observées au stade $J_{3}$. Le rapport des $i m$ moyens de type 1 et de type 2 $(2,18)$ est de l'ordre du rapport des im moyens du stade $A$ et du stade $J_{3}(2,77)$. Ceci signifierait qu'il y a accouplement entre bordés au stade $A$, pesant $8-13 \mathrm{~g}$ environ, et bordés au stade $\mathrm{J}_{3}$, de $7-12 \mathrm{~g}$, donc plus petits.

Dans les 26 couples observés au hasard pendant la période de reproduction d'autres générations $F_{1}$, le poids moyen des glandes à albumen de type 1 est de $1046 \mathrm{mg} \pm 292\left(i m_{1}=9,86 \pm 1,83\right)$, tandis que le poids moyen des glandes de type 2 atteint seulement $460 \mathrm{mg} \pm 220\left(\mathrm{im}_{2}=\right.$ $4,60 \pm 1,83)$, le rapport des im $(2,14)$ étant identique à celui observé pour les 10 couples précédents.

Dans le lot des géniteurs (parents de $F_{1}$ ) (tableau I), des accouplements ont lieu dans les premiers jours de la remise en activité après hibernation. Les couples sont aussi formés d'un animal de type 1 et d'un animal de type 2, les tractus génitaux étant comparables à ceux de la fig 4 . Le poids moyen des glandes à albumen est cependant inférieur à celui observé dans la génération $F_{1}$, probablement à la suite du jeûne subi pendant l'hibernation. Dans d'autres lots d'adultes remis en activité après hibernation, on note le même phéno- 


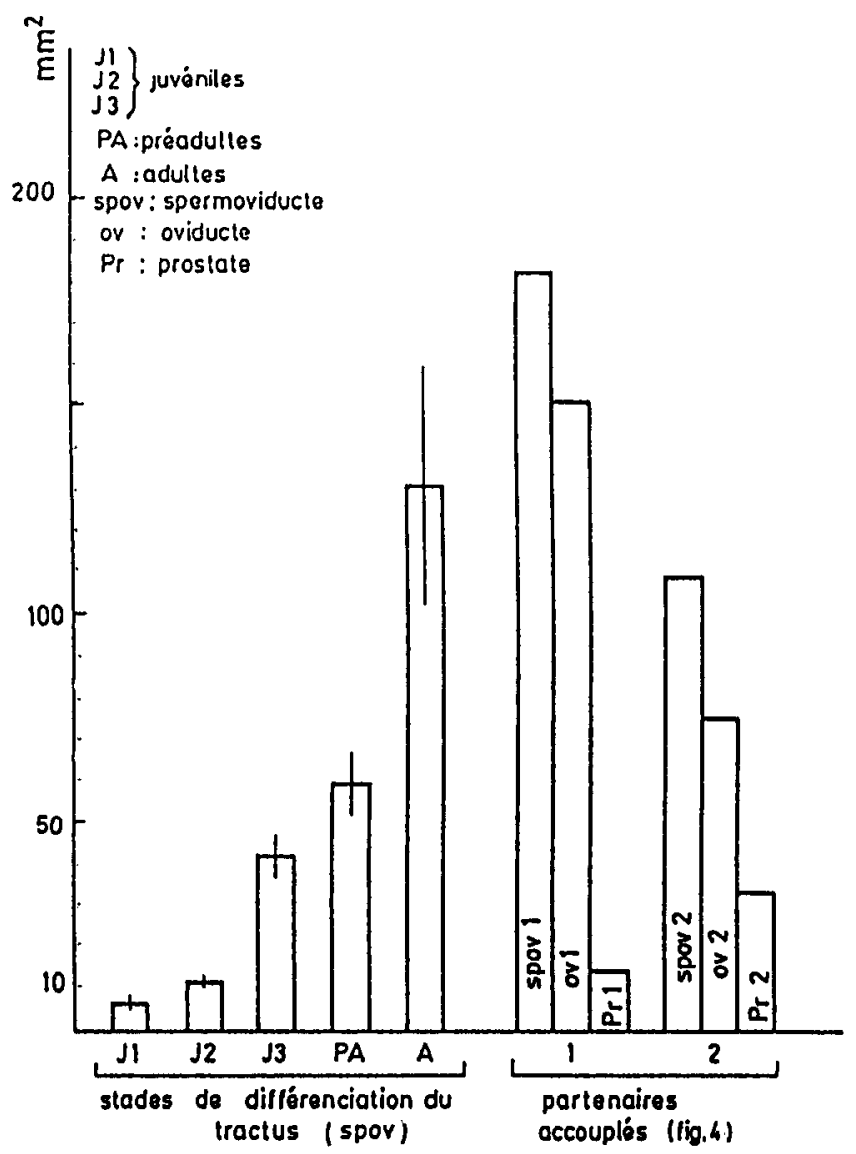

Fig 5. Analyse quantitative de l'évolution de la surface de la section transversale du spermoviducte.

mène : $i m_{1}=9,46 \pm 3,19$ et $i m_{2}=2,85 \pm$ 1,03 (3 couples), $i m_{1}=6,10 \pm 0,72$ et $i m_{2}=$ $3,45 \pm 1,48$ (5 couples).

Au moment de la ponte

Avant la ponte (AP, fig 3), chez les escargots n'ayant pas commencé à former leurs œufs, le poids moyen de la glande à albumen n'est pas significativement différent par rapport aux glandes des accouplés de type 1, ni par rapport aux glandes les plus évoluées dans la classe de poids de 1011 g. Par contre, le poids moyen de la glande à albumen des copulants de type 2 a augmenté significativement entre le premier accouplement et la ponte, si bien qu'il n'existe plus qu'une catégorie de glandes à albumen. Après la ponte (post-ponte, PP, fig 3), le poids et l'indice moyen diminuent de moitié et l'augmentation des écarts types par rapport à la période avant-ponte indique que l'irrégularité de la glande à albumen est réapparue. Dans d'autres lots d'escargots élevés de la même manière, des valeurs identiques ont été observées avant la formation des œufs (poids $=1280$ 
$\mathrm{mg} \pm 156 ; i m=12,18 \pm 1,78 ; n=13$ animaux) et après la ponte (poids $=490 \mathrm{mg} \pm$ 245; im = 4,95 $\pm 2,29 ; n=7$ animaux).

\section{Mesure de l'activité glandulaire des organes accessoires}

\section{Étude morphométrique et pondérale du spermoviducte et de ses composants}

L'évolution de la surface transversale de la partie moyenne du spermoviducte (fig 5) met en évidence une augmentation importante de son développement entre le stade $J_{2}$ et le stade $J_{3}$, dans la période qui correspond à la formation du rebord de la coquille, et ensuite entre le stade PA et le stade A. À l'accouplement, l'importance de la surface transversale de l'oviducte du type 1 par rapport à celle de la prostate montre que l'hyperdéveloppement du spermoviducte est essentiellement causé par l'épaississement glandulaire de la paroi de l'oviducte. Par contre, la surface transversale de la prostate est plus importante chez le type 2 dont la maturation sexuelle femelle est moins avancée.

La différence de poids entre les spermoviductes (spov) 1 et 2 n'est pas statistiquement significative (fig 6), mais néanmoins, les résultats de l'étude pondérale des composants du spov indiquent que les différences relatives entre les tractus des 2 partenaires sont en relation avec le développement prépondérant de l'oviducte (ov) pour le type 1, et l'importance de la prostate pour le type 2.

\section{Dosage du galactogène dans la glande à albumen (fig 7)}

Le galactogène présent dans la glande à albumen à partir du stade $\mathrm{J}_{2}$ augmente pendant le développement des animaux pour atteindre une moyenne de $76,4 \mathrm{mg}$ par glande à la fin de la croissance chez les escargots adultes (A). Au premier ac- couplement, la quantité moyenne de galactogène dans les glandes les plus développées de type 1 est 4 fois plus importante que celle dosée dans les glandes de type 2 , mais n'est pas significativement différente par rapport aux animaux ayant un tractus génital au stade $A$ en fin de croissance dans la classe de poids de 10-11 g. Chez les types 2, la quantité moyenne de galactogène n'est pas significativement différente par rapport au stade $J_{3}$ et augmente de 4,5 fois entre le premier accouplement et la ponte où les glandes à albumen contiennent en moyenne $103 \mathrm{mg}$ de galactogène. En comparaison avec le

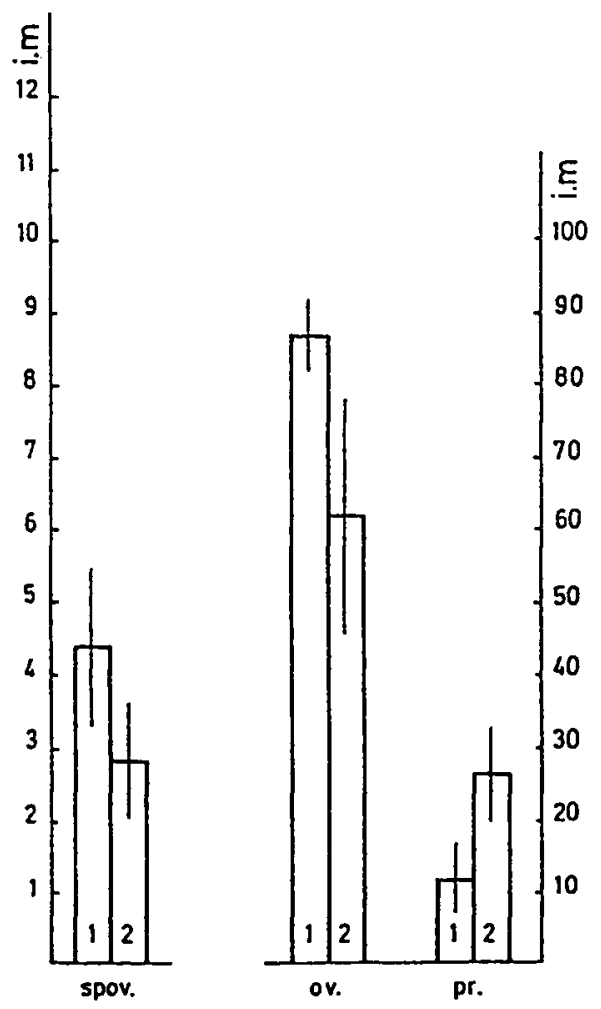

Fig 6. Comparaison des indices de maturation (im) du spermoviducte (spov), de l'oviducte (ov) et de la prostate (pr) des partenaires (1 et 2) au premier accouplement. 


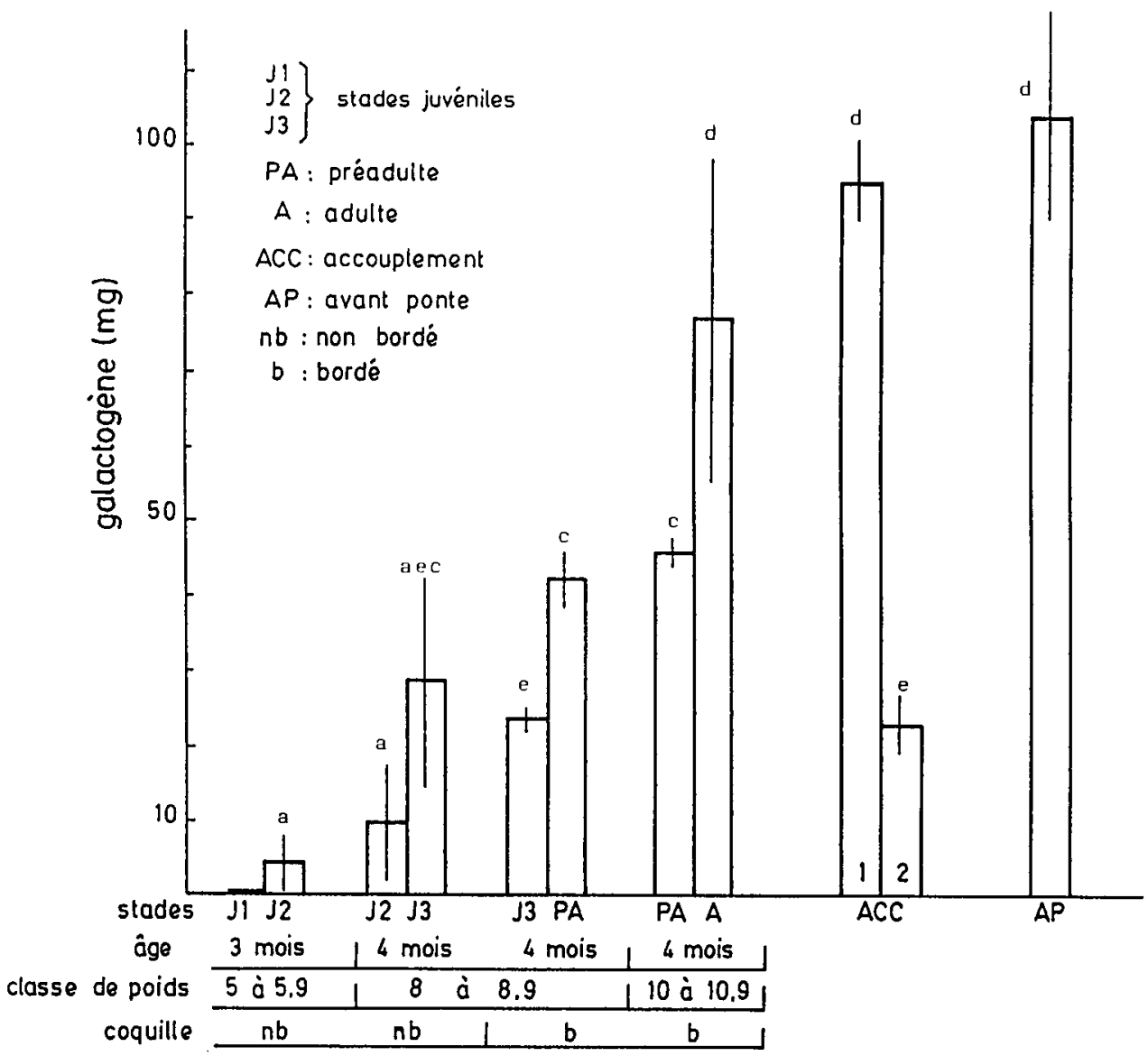

Fig 7. Comparaison de la quantité de galactogène contenue dans la glande à albumen à différents stades de la croissance et de la reproduction. Les groupes portant des lettres différentes sont significativement différents entre eux.

diagramme de la fig 3 , le contenu de galactogène de la glande à albumen paraît en relation avec l'indice de maturation glandulaire.

\section{DISCUSSION}

Dans des conditions constantes et favorables d'élevage en laboratoire, une importante hétérogénéité est mise en évidence au cours de l'évolution d'une population
d'Helix aspersa, aussi bien dans la croissance pondérale des animaux que dans la maturation sexuelle caractérisée par l'asynchronisme du développement du tractus génital.

\section{Période de croissance}

Dans les histogrammes des classes de poids à 3 mois et 4 mois, la présence de 2 pics dans la répartition des animaux indi- 
que que l'ensemble renfermerait des individus de 2 sous-ensembles. La population paraît constituée, d'une part d'escargots à croissance rapide atteignant un poids élevé au moment de la formation du bord de la coquille qui indique le début du stade adulte, d'autre part d'escargots qui grandissent lentement et arrivent à l'état adulte avec un poids plus faible et plus tardivement. La croissance aboutit ainsi à des adultes de poids inégaux et dont le début de la maturité sexuelle s'étale du $3^{e}$ au $4^{e}$ mois. Le facteur responsable de l'hétérogénéité de la population ne peut être attribué aux conditions d'environnement qui sont uniformes. Cependant, le fait que la population étudiée soit une première génération $\left(F_{1}\right)$ issue de plusieurs escargots prélevés dans la nature pourrait être un facteur d'hétérogénéité car il a été démontré que les différences liées à l'habitat naturel plus ou moins favorable persistent dans la croissance et les processus de reproduction du Basommatophore Lymnaea peregra jusqu'à la deuxième génération (Calow, 1981).

Des différences de croissance et de maturation sexuelle ont aussi été remarquées chez différentes espèces de Stylommatophores : Arion ater (Smith, 1966), Ariolimax reticulatus (Runham et Laryea, 1968), Euparypha pisana (LazaridouDimitriadou, 1978), Arianta arbustorum (Baur et Rabaud, 1988) et Limax maximus chez qui Prior (1983) considère que ces variations impliqueraient plutôt une hétérogénéité génétique que des conditions d'environnement non uniformes.

Dans le cas d'Helix aspersa, nous suggérons que l'hétérogénéité de croissance et de différenciation sexuelle correspond à une stratégie de groupe qui implique des influences interindividuelles. En effet, lorsque les individus en croissance d'une population hétérogène d'Helix aspersa de même âge sont triés par classes de poids (homogénéisation artificielle), il réapparaît des escargots à croissance lente et des escargots à croissance rapide dans chaque lot, et ceci plusieurs fois au cours des tris successifs (Laurent et al, 1984). Le mécanisme de ces intéractions entre individus n'est pas connu, mais il est confirmé par les expériences d'élevage mettant en évidence l'influence de la densité de la population (Lucarz et Gomot, 1984, 1985).

Des études antérieures (Abeloos, 1944; Lüsis, 1961; Gomot et Enée, 1980) donnaient une idée générale du développement du tractus génital en fonction de la croissance des Pulmonés. L'analyse détaillée de l'appareil génital de chaque classe d'âge et de poids d'Helix aspersa permet de déceler l'existence de deux sous-ensembles de même importance quant au développement de la glande à albumen et de l'oviducte. Les escargots les plus gros à croissance plus rapide atteignent le plus tôt la maturité femelle et constituent les premiers individus de type 1. On peut penser aussi que les ancêtres des Helix auraient été des espèces dioïques, où les femelles auraient eu une taille supérieure. Après avoir acquis l'hermaphrodisme, les escargots auraient maintenu un caractère lié au sexe, responsable de la croissance différentielle, exprimée par un tractus génital qui se développerait chez la moitié d'entre eux dans le sens femelle, et chez l'autre moitié dans le sens mâle. Cela permettrait de comprendre la raison de la variabilité de la croissance (2 pics) et la difficulté d'établir des critères d'homogénéité sur des classes d'âge-poids dans ces ensembles hétérogènes.

\section{Période de reproduction}

Au moment de la reproduction, la rencontre des partenaires, adultes toujours bordés, ne se fait pas au hasard, puisqu'au 
moment du premier accouplement, on observe une différence importante du développement du tractus génital des 2 copulants de chaque couple. Chez l'animal de type 1 , le plus évolué, l'hyperdéveloppement du tractus femelle est concomitant de la vidange de la sécrétion prostatique mâle (Enée, 1984). Chez le type 2, le poids de la glande à albumen, son faible contenu en galactogène, l'hyperdéveloppement de la prostate indiquent que le tractus génital est au stade $J_{3}$ à dominante mâle (fig 3). Cette disparité sexuelle anatomique à l'accouplement entre 2 adultes est probablement la conséquence de l'asynchronisme sexuel observé pendant la croissance; elle se traduit par une différence de comportement qui permet à 2 animaux à des stades physiologiques différents de se reconnaître. Cette différence de maturité sexuelle des copulants apparaît comme un fait majeur car il a été confirmé dans d'autres lots, aussi bien au début de la remise en activité d'adultes après hibernation que pendant la première période de reproduction de plusieurs groupes. La disparité du développement du tractus génital à l'accouplement peut donc être considérée comme faisant partie d'une stratégie de reproduction chez Helix aspersa, mais il n'a pas été possible d'élucider un comportement comme mâle ou femelle en raison d'un orifice génital unique et de l'échange réciproque de spermatophores; on ne peut donc pas distinguer un individu femelle et un individu mâle comme chez Lymnaea stagnalis qui possède un orifice mâle et un orifice femelle distincts et où le mâle adopte un comportement femelle au deuxième accouplement (Van Duivenboden et Ter Maat, 1985). Le devenir des escargots de type 1 ou 2 entre le premier accouplement et la ponte n'a pas été spécialement étudié, mais nous avons observé en général que 1 ou 2 jours après l'accouplement, l'un des copulants pond, probablement l'animal de type 1 le plus évolué, et que l'autre (type 2) se réaccouple au moins une fois avant de pondre.

Après la ponte, l'escargot dont l'appareil génital a régressé au type 2 après avoir produit les couches périvitellines des œufs, se réaccouple dans les 2 à 5 j avec un individu de type 1. Des études statistiques sont cependant nécessaires pour affirmer que laccouplement agirait, d'une part comme un stimulus à court terme pour l'individu de type 1 dont la sécrétion d'hormone de ponte serait probablement activée chez cet animal physiologiquement prêt à pondre, d'autre part comme un stimulus à long terme sur l'évolution femelle du tractus de l'individu de type 2. En accord avec cette hypothèse, l'observation d'accouplements répétés avant la ponte pendant un cycle de 7 semaines (Madec et Daguzan, 1987; Bonnefoy-Claudet et al, 1987) suggère une corrélation entre la copulation et la stimulation du développement du tractus femelle qui atteint son maximum à la ponte, alors que l'asynchronisme disparait. De plus, la stimulation de la synthèse de galactogène de la glande à albumen après la fin de l'accouplement (Bride et al, 1991) indique que celui-ci joue un rôle dans l'augmentation rapide du contenu en galactogène des glandes de type 2 entre le premier accouplement et la ponte. Ceci est en accord avec les observations réalisées chez d'autres espèces. Chez Lymnaea stagnalis, le premier effet de la copulation est mis en évidence sur la stimulation du développement des organes accessoires femelles par le transfert de sperme (Van Duivenboden, 1983). Chez Arianta arbustorum, l'influence stimulante de l'accouplement est démontrée par rapport aux individus isolés par l'augmentation de la taille des pontes après copulations successives (Baur, 1988). Le mode d'action de l'accouplement sur les processus de reproduction a fait l'objet d'études expérimentales chez Lymnaea stagnalis, Heli- 
soma duryi, Helix aspersa. II implique, d'une part l'injection par le dard (Adamo et Chase, 1988) d'une phéromone de la glande du dard (Chung, 1986), et d'autre part le transfert, entre les 2 partenaires, de sperme qui peut avoir 2 effets opposés selon le stade de développement des animaux (Van Duivenboden, 1983; Van Duivenboden et al, 1986). Accompagnant le sperme, des sécrétions des organes accessoires mâles contenant des prostaglandines (Kunigelis et Saleuddin, 1986) et des substances apparentées aux peptides et neuropeptides des centres gonadotropes femelles peuvent agir comme phéromones endogènes (Van Minnen et Vreugdenhil, 1987). Des études supplémentaires sont nécessaires chez Helix aspersa pour préciser si l'action des susbtances échangées à l'accouplement est directe sur l'évolution du tractus génital femelle ou indirecte, par l'activation de centres endocrines (Mukai et Saleuddin, 1989; Saleuddin et al, 1983, 1989).

En conclusion, ce travail complète l'étude de Gomot et Enée (1980) qui donnait seulement une approximation du rapport entre l'évolution morphologique du tractus et les différentes périodes de la croissance des animaux. Parmi les éléments nouveaux les plus importants, il faut remarquer que :

- le développement individuel d'Helix aspersa au sein d'une population se fait selon 2 taux de croissance différents, le plus élevé donnant des escargots plus gros (poids) qui présentent un mûrissement précoce de type femelle, alors que les autres aboutissent à des adultes au stade mâle;

- les copulants présentent un dimorphisme sexuel interne très accentué (partenaires de type 1 à dominante femelle, de type 2 à dominante mâle);

- l'état adulte ne peut être défini avec certitude en terme d'indice de maturation, les
$J_{3}$ présentant un développement du tractus génital identique à celui des adultes à dominante mâle (copulants de type 2), les A correspondant seulement aux adultes à dominante femelle (copulants de type 1);

- l'asynchronisme du développement de l'appareil génital apparaît comme une stratégie caractéristique de l'évolution de la population chez Helix aspersa;

- la disparition de la différence entre les appareils génitaux entre l'accouplement et la ponte suggère un rôle régulateur de l'accouplement.

\section{REMERCIEMENTS}

Les auteurs remercient $\mathbf{B}$ Jolibois pour la préparation du manuscrit.

\section{RÉFÉRENCES}

Abeloos M (1944) Recherches expérimentales sur la croissance. Bull Biol Fr Belg 78, 215256

Adamo SA, Chase R (1988) Courtship and copulation in the terrestrial snail Helix aspersa. Can J Zool 66, 1446-1453

Baur B (1988) Repeated mating and female fecondity in the simultaneously hermaphroditic land snail Arianta arbustorum. $J$ Invertebr Reprod Dev 14, 197-204

Baur B, Rabaud C (1988) Life history of the land snail Arianta arbustorum along an altitudinal gradient. J Anim Ecol 57, 71-87

Bonnefoy-Claudet R, Deray A (1987) Modalités de reproduction de l'escargot Helix aspersa maxima en fonction de la photophase; comparaison avec Helix aspersa aspersa. Haliotis 16, 69-75

Bonnefoy-Claudet R, Deray A, Griffond B (1987) La reproduction de l'escargot Helix aspersa Müller en fonction des conditions d'environnement. Données synthétiques. Haliotis 16, 57-67

Bride J, Gomot L, Saleuddin ASM (1991) Mating and 20 hydroxyecdysone cause increased 
galactogen synthesis in the albumen gland of Helix aspersa (Mollusca). Comp Biochem Physiol 821B (sous presse)

Calow $P$ (1981) Adaptational aspects of growth and reproduction in Lymnaea peregra (Gastropoda: Pulmonata) from exposed and sheltered aquatic habitats. Malacologia 21, 5-13

Calow P (1983) Life cycle patterns and evolution. In: The Mollusca (Russell-Hunter WD, ed), vol 6, Ecology, Acad Press, London, 649-678

Charrier M (1980) Contribution à la biologie et à l'écophysiologie de l'escargot Petit-Gris, Helix aspersa Müller (Gastéropodes Pulmonés Stylommatophores). Thèse Doctorat $3^{e}$ cycle, Univ Rennes I

Chung DJD (1986) Stimulation of genital eversion in the land snail Helix aspersa by extracts of the glands of the dart apparatus. $J$ Exp Zool 238, 129-139

Courtot AM, Gomot L (1982) Les stades de l'organogenèse et la cytodifférenciation de la glande à albumen de l'escargot Helix aspersa Müller. Reprod Nutr Dév 22, 666-680

Enée J (1984) Contribution à l'étude de la croissance de l'escargot Helix aspersa. Ontogenèse de l'appareil génital et recherche cytologique sur la différenciation et la sécrétion de la prostate. Thèse Doctorat $3^{e}$ cycle, Univ Besançon

Enée J, Griffond B (1983) Étude histologique et ultrastructurale de l'appareil génital postembryonnaire d'Helix aspersa Müller. Reprod Nutr Dév 23, 927-941

Enée J, Gomot L (1984) Organogenèse et cytodifférenciation de la prostate de l'escargot Helix aspersa Müller. Reprod Nutr Dév 24, 563-585

Enée J, Bonnefoy-Claudet R, Gomot L (1982) Effet de la photopériode artificielle sur la reproduction de l'escargot Helix aspersa Müll. CR Séances Acad Sci Paris 294, 357-360

Geraerts WPM, Joosse J (1984) Freshwater snails (Basommatophora). In: The Mollusca (AS Tompa, NH Verdonk, JAM Van Biggelaar, eds), vol 7, Acad Press, New York, 142208

Gomot L, Enée J (1980) Biologie de la reproduction de l'escargot Helix aspersa Müll: les phases de croissance et la différenciation sexuelle. Atti Acad Fisiocritici Siena, 73-85
Gomot L, Deray A (1987) Les escargots. Recherche 18, 302-311

Gomot L, Enée J, Laurent J (1982) Influence de la photopériode sur la croissance pondérale de l'escargot Helix aspersa Müller en milieu contrôlé. CR Séances Acad Sci Paris 294, 749-752

Goodfriend GA (1986) Variation in land-snail shell form and size and its causes: a review. Syst Zool 35, 204-223

Joosse J, Geraerts WPM (1984) Endocrinology. In: The Mollusca (ASM Saleuddin, KM Wilbur, eds), vol 4, Physiology, Acad Press, New York, 317-406

Joosse J, Van Elk R (1986) Trichobilharzia ocellata physiological characterization of giant growth, glycogen depletion and absence of reproductive activity in the intermediate snail Lymnaea stagnalis. Exp Parasitol 62, 1-3

Kunigelis SC, Saleuddin ASM (1986) Reproduction in the freswater gastropod, Helisoma: involvement of prostaglandin in egg production. Int $J$ Invert Reprod Dev 10, 159-167

Laurent J, Deray A, Grimard AM (1984) Influence de la photopériode, du degré d'hétérogénéité de la population sur la dynamique de croissance et la maturation sexuelle de l'Escargot Helix aspersa. CR Séances Soc Biol Fil 178, 421-441

Lazaridou-Dimitriadou M (1978) Contribution à l'écophysiologie d'un Gastéropode Pulmoné dunicole Euparypha pisana (Müller) du littoral armoricain. Thèse Doctorat $3^{e}$ cycle, Univ Rennes

Lucarz A, Gomot L (1984) Étude expérimentale de l'effet du groupement sur la ponte d'Helix aspersa Müller. Intern J Invertebr Reprod Dev 7, 185-192

Lucarz A, Gomot L (1985) Influence de la densité de population sur la croissance diamétrale et pondérale de l'escargot Helix aspersa Müller dans différentes conditions d'élevage. $J$ Molluscan Stud 51, 105-115

Lüsis O (1961) Post-embryonic changes in the reproductive system of the slug Arion ater rufus $L$. Proc Zool Soc Lond 137, 433-468

Madec L, Daguzan J (1987) Étude de quelques facteurs affectant la reproduction de l'escargot Petit-Gris Helix aspersa Müller. Haliotis 16, 77-91 
Mukai ST, Saleuddin ASM (1989) Mating increases the synthetic activity of the neurosecretory caudodorsal cells of Helisoma duryi (Mollusca: Pulmonata). Can J Zool 67, 2363-2367

Prior $D$ (1983) The relationship between age and body size of individuals in isolated clutches of terrestrial slug, Limax maximus (Linnaeus, 1858). J Exp Zool 225, 321-324

Runham NW, Laryea AA (1968) Studies on the maturation of the reproductive system of Agriolimax reticulatus (Pulmonata: Limacidae). Malacologia 7, 13-108

Saleuddin ASM, Kunigelis SC, Schollen LM, Breckenridge WR, Miksys SL (1983) Studies on endocrine control of reproduction in Helisoma and Helix. In: Molluscan NeuroEndocrinology (Lever J, Boer $\mathrm{HH}$, eds), North Holland Publ Co, Amsterdam, 138-142

Saleuddin ASM, Ashton ML, Khan HR (1989) Mating induced release of granules by the endocrine dorsal body cells of the snail Helisoma duryi (Mollusca). J Exp Zool 250, 206213

Smith BH (1966) Maturation of the reproductive tract of Arion ater (Pulmonata: Arionidae). Malacologia 4, 325-349
Sokal RR, Rolf FJ (1969) Biometry. Freeman, Londres

Tompa BJ (1984) Land snails (Stylommatophora). In: The Mollusca (Wilbur KM, ed) vol 7. Reproduction, Acad Press, New-York, 48140

Van Duivenboden YA (1983) Transfer of semen accelerates the onset of egg-laying in female copulants of the hermaphrodite freshwater snail, Lymnaea stagnalis. Intern $J$ Invertebr Reprod 6, 249-257

Van Duivenboden YA, Ter Maat A (1985) Masculinity and receptivity in the hermaphrodite pond snail Lymnaea stagnalis. Anim Behav 33, 885-891

Van Duivenboden YA, Pieneman AW, Ter Maat A (1986) Multiple mating suppresses fecundity in the hermaphrodite freshwater snail $L y m$ naea stagnalis. Anim Behav 33, 1184-1191

Van Handel E (1965) Estimation of glycogen in small amount of tissue. Anal Biochem 11, 256-265

Van Minnen J, Vreugdenhil E (1987) The occurrence of gonadotropic hormones in the central nervous system and reproductive tract of Lymnaea stagnalis. An immunocytochemical and in situ hybridization study. In: Neurobiology. Molluscan Models (Boer HH, Geraerts WPM, Joosse J, eds), 62-68 